\title{
Over Approximate Flow Pipeline Volume Algorithm for Three Variables Real-Time Linear Algebraic Hybrid Automaton
}

\author{
Hao Yang \\ 415360889@qq.com \\ College of New Media, Zhejiang University Of Media and Communications, China
}

\begin{abstract}
Keywords: Hybrid automata; Algebraic program; Volume algorithm; Flow pipeline
\end{abstract}
\begin{abstract}
In order to compute trajectory of three variables real-time linear algebraic Hybrid Automaton, three dimensional over approximate flow pipeline volume algorithm for three variables real-time linear algebraic Hybrid Automaton is proposed. Firstly, it defines the three variables real-time linear algebraic program and three variables real-time linear algebraic Hybrid Automaton. And then, it introduces flow pipe approximate method and gives three conclusions of continuous transition relation. Next it obtains three dimensional over approximate flow pipeline volume algorithm. Finally, fighter flight movement automaton example proves algorithm validation.
\end{abstract}

\section{Introduction}

In the reachable set computation, it is able to ensure that computation reachable set is expansion of actual reachable set. This type of verification method is over approximation method. For security verification problems, over approximate method is from initial state set and forward calculation reachable set. Finally, it checks whether reachable set within security states set or not. Over approximate method compute state value along system dynamic evolution[1,2]. Continuous state space is divided into finite subspaces. It uses approximate method to judge all reachable subspaces. It obtains possibility discrete transition[3].

The reachable set expression method and continuous dynamic type limit are two key questions in the over approximate method. The reachable set expression method has hyper rectangle method, polyhedron method and ellipsoid method. Hyper rectangle method is easy to express. Hyper rectangle surface number and continuous space dimension become the linear relations. In order to make over approximate not excessively conservative, it needs increase the massive grids. Polyhedron method can be done very good approximation in convex sets[4]. But the number of polyhedron surfaces and apexes increases with continuous space dimension. Polyhedron method is not suitable for the high dimensional system. Ellipsoid and continuous space dimension become secondary relation[5]. But intersect and merge parts no longer are ellipsoids. It is seen that reachable set expression more complex, the more hyper rectangle, polyhedron and ellipsoid need. At present most over approximate method is difficult to deal with nonlinear system and it is generally limited continuous dynamic behaviour to linear systems.

This paper is organized as follows. In section 2, it introduces three variables real-time linear algebraic programs and establishes three variables real-time linear algebraic Hybrid Automaton. In section 3, it proposes the flow pipeline over approximate method of three variables real-time linear algebraic Hybrid Automaton. In section 4, it gets three dimensional over approximate flow pipeline volume algorithm of three variables real-time linear algebraic Hybrid Automaton. In section 5, fighter flight movement automaton example proves algorithm validation.

\section{Three Variables Real-Time Linear Algebraic Hybrid Automata}

Definition 1(Three variables real-time linear algebraic program) Let $\mathrm{R}$ be the set of real numbers, $x_{i}(i=1,2,3)$ and $x_{i}^{\prime}(i=1,2,3)$ be the real variables, $t \in \mathrm{R}$ be the time variable. A three variables real-time linear algebraic program is an algebraic program likes $X^{\prime}=X+F(t)$, where $X^{\prime}=\left(x_{1}^{\prime}, x_{2}^{\prime}, x_{3}^{\prime}\right)^{\mathrm{T}}$ is the post-state value of three variables real-time linear algebraic program transition, 
$X=\left(x_{1}, x_{2}, x_{3}\right)^{\mathrm{T}}$ is the pre-state value of three variables real-time linear algebraic program transition. $F(t)=\left(f_{1}(t), f_{2}(t), f_{3}(t)\right)^{\mathrm{T}}$ is three dimensional column vector. $f_{i}(t)$ is a variable polynomial of degree one which the constant term is zero or $f_{i}(t)$ is zero. $f_{i}(t)(i=1,2,3)$ is monotonous and at least includes a polynomial of degree one.

Definition 2(Three Variables Real-Time Linear Algebraic Hybrid Automaton) A three variables real-time linear algebraic Hybrid Automaton is a tuple $\mathrm{H}=\langle\mathrm{Q}, \mathrm{V}, \mathrm{HX}$, Init, Lab, E, Inv, F, R $>$, where

1) $Q$ is a set of system discrete locations.

2) $\mathrm{V}$ is a set of system continuous variables.

3) $\mathrm{HX}$ is a set of system continuous variables values.

4) Init $\in Q \times H X$ is a set of system initial states. System initial states value set $Y_{0} \subseteq H X$. $Y_{0}$ is a convex polygon.

5) Lab is a set of discrete transition programs. A discrete transition programs is a linear algebraic program $X^{\prime}=A X$ or $X^{\prime}=A X+b$.

6) $\mathrm{E}$ is a set of discrete transitions.

7) Inv is a set of continuous variables invariants.

8) $\mathrm{F}$ is a set of three variables real-time linear algebraic programs which describe system continuous variables dynamic processes.

9) $\mathrm{R}$ is a set of discrete location transition conditions.

\section{Flow Pipeline over Approximate Method}

In the three variables real-time linear algebraic Hybrid Automaton, system continuous dynamic behaviour trajectory in any time period $\left[t_{0}, t_{f}\right]$ cannot be accurately calculated. In order to effectively compute system continuous dynamic reachable set from system initial state $<q_{0}, X_{0}>$. It uses flow pipeline over approximate method to analyze continuous transition relation of three variables real-time linear algebraic Hybrid Automaton.

Let $\left\langle q_{i}, X_{i}\right\rangle$ be an invariant initial state of three variables real-time linear algebraic Hybrid Automaton, $Y_{i}$ be system invariant initial state value set. The system reachable set which is from initial state $\left\langle q_{i}, X_{i}\right\rangle$ and after $\mathrm{t}$ time continuous transition is defined as $R_{t}\left(Y_{i}\right)=\left\{X_{t} \mid X_{t}=X\left(t, X_{i}\right), \exists X_{i} \in Y_{i}\right\}$.

Definition 3(Flow Pipeline) Reachable set which is from initial state $\left.<q_{i}, X_{i}\right\rangle$ in an invariant set of three variables real-time linear algebraic Hybrid Automaton and in any time period $\left[t_{0}, t_{f}\right]$ is called flow pipeline. It defined as $R_{\left[t_{0}, t_{f}\right]}\left(Y_{i}\right)=\bigcup_{t \in\left[t_{0}, t_{f}\right]} R_{t}\left(Y_{i}\right)$.

Flow pipeline is from initial state $<q_{0}, X_{0}>$ of three variables real-time linear algebraic Hybrid Automaton in each time period reachable state set. Flow pipeline can reflect system continuous variables value change in one time period and describe system continuous dynamic evolution process. The over approximate flow pipeline $\hat{R}_{\left[t_{0}, t_{f}\right]}\left(Y_{i}\right)$ in the time period $\left[t_{0}, t_{f}\right]$ is union of $\mathrm{N}$ segments flow pipeline $\hat{R}_{\left[t_{k-1}, t_{k}\right]}\left(Y_{i}\right)(k=1,2, \cdots, N)$. It can be written as

$$
\hat{R}_{\left[t_{0}, t_{f}\right]}\left(Y_{i}\right)=\bigcup_{k=1}^{N} \hat{R}_{\left[t_{k-1}, t_{k}\right]}\left(Y_{i}\right), \forall k, R_{\left[t_{k-1}, t_{k}\right]}\left(Y_{i}\right) \subseteq \hat{R}_{\left[t_{k-1}, t_{k}\right]}\left(Y_{i}\right) .
$$




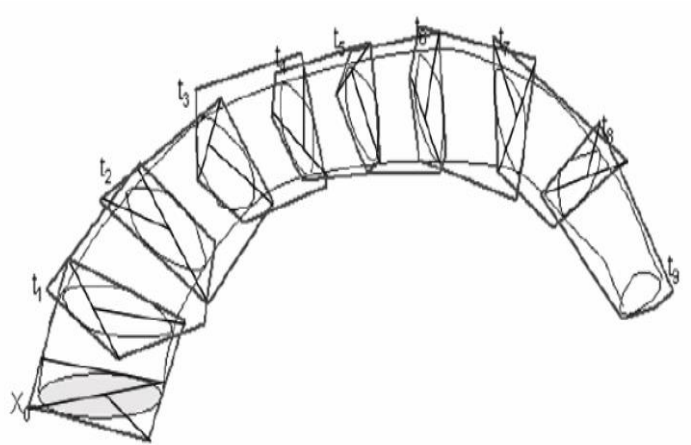

Figure 1. Flow pipeline section over approximation

\section{Three Dimensional over Approximate Flow Pipeline Volume Algorithm}

Three dimensional flow pipeline volume algorithm is based on three dimensional convex polyhedron volume algorithm. Three dimensional convex polyhedron volume algorithm is usually adopted segmentation method. Firstly, it divides three dimensional convex polyhedron into some three dimensional tetrahedrons. Secondly, it computes every three dimensional tetrahedron volume. Finally, it adds all tetrahedron volume into three dimensional convex polyhedron volume.

Assume that a three dimensional convex polyhedron can be divided into $\mathrm{m}$ three dimensional tetrahedrons. The points of $\mathrm{m}$ three dimensional tetrahedrons are $P_{0}^{i}, P_{1}^{i}, P_{2}^{i}, P_{3}^{i}$, where $i=1, \cdots, m$. The coordinate of vertex $P_{j}^{i}$ is $\left(x_{j, 1}^{i}, x_{j, 2}^{i}, x_{j, 3}^{i}\right)$, where $i=1, \cdots, m$ and $j=0,1,2,3$. The three dimensional convex polyhedron volume $\mathrm{V}$ is the sum of $\mathrm{m}$ three dimensional tetrahedral volumes. $V=\sum_{i=1}^{m} V_{i}=\sum_{i=1}^{m} \frac{1}{6}\left|\operatorname{det}\left(P_{1}^{i}-P_{0}^{i} \quad P_{2}^{i}-P_{0}^{i} \quad P_{3}^{i}-P_{0}^{i}\right)\right|$.

The three dimensional over approximate flow pipeline $\hat{R}_{[k T,(k+1) T]}\left(Y_{i}\right)$ volume algorithm in the time period $[k T,(k+1) T]$ is listed as follows:

1) Initial. $t_{0}=k T, t_{f}=(k+1) T$.

2) In the time period $\left[t_{0}, t_{f}\right]$, through m vertexes $v_{1}(0), v_{2}(0), \cdots, v_{m}(0)$ of three variables real-time linear algebraic Hybrid Automaton in current invariant set initial states value set $Y_{i}$ and corresponding three variables real-time linear algebraic program, we get $\mathrm{m}$ corresponding vertexes $v_{1}\left(t_{0}\right), v_{2}\left(t_{0}\right), \cdots, v_{m}\left(t_{0}\right)$ in time $t_{0}$ and m corresponding vertexes $v_{1}\left(t_{f}\right), v_{2}\left(t_{f}\right), \cdots, v_{m}\left(t_{f}\right)$ in time $t_{f}$. 3) It uses $m$ corresponding vertexes $v_{1}\left(t_{0}\right), v_{2}\left(t_{0}\right), \cdots, v_{m}\left(t_{0}\right)$ in time $t_{0}$ and $m$ corresponding vertexes $v_{1}\left(t_{f}\right), v_{2}\left(t_{f}\right), \cdots, v_{m}\left(t_{f}\right)$ in time $t_{f}$ to compute three dimensional flow pipeline volume $V . V_{\left[t_{0}, t_{f}\right]}=\bigcup_{k=1}^{N} V_{\left[t_{k-1}, t_{k}\right]}$ is the over approximate flow pipeline volume $\hat{R}_{\left[t_{0}, t_{f}\right]}\left(Y_{i}\right)$ of flow pipeline $R_{\left[t_{0}, t_{f}\right]}\left(Y_{i}\right)$ in the time period $\left[t_{0}, t_{f}\right]$.

In the discrete location $q_{j}, V L_{j}$ represents over approximate flow pipeline volume of three variables real-time linear algebraic Hybrid Automaton. After system leave discrete location $q_{j}$, the front $j+1$ discrete locations over approximate flow pipeline volume sum is $V_{j} \cdot V_{j}=V L_{0}+V L_{1}+\cdots+V L_{j}$.

\section{Experiments}

Fighter is a common military weapon in modern warfare and plays an important role on the outcome of the war. It uses space rectangular coordinate system o-xyz to describe flight trajectory of the fighter. $\mathrm{x}$ axis represents left and right space. $y$ axis represents front and rear space. $z$ axis represents upper and lower space. The coordinate of object in the space rectangular coordinate system 0 -xyz is 
$X=\left(x_{1}, x_{2}, x_{3}\right)^{\mathrm{T}} \cdot x_{1}, x_{2}, x_{3}$ are respectively represent the values in the $\mathrm{x}$ axis, $\mathrm{y}$ axis and $\mathrm{z}$ axis. Coordinate values of fighter change along with the time $t$.

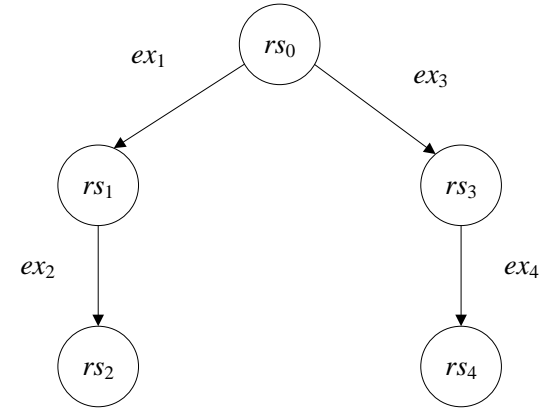

Figure 2. Fighter flight movement automaton

In Figure 3, there is a fighter flight movement automaton. $\mathrm{rs}_{0}$ is the initial node of three variables real-time linear algebraic Hybrid Automaton. Initial node has the set Init= $\mathrm{S}_{0}$ of initial state values. In the node $\mathrm{rs}_{0}$, fighter flights parallel constant speed. In the node $\mathrm{rs}_{1}$ and node $\mathrm{rs}_{4}$, fighter flights rising constant speed. In the node $\mathrm{rs}_{2}$ and node $\mathrm{rs}_{3}$, fighter flights falling constant speed.

The set of object motion automaton discrete locations is $Q=\left\{q_{0}, q_{1}, q_{2}, q_{3}, q_{4}\right\}$. The set of automaton initial sates is $S_{0}=\left\{q_{0}\right\} \times\left\{\left(0 \leq x_{1} \leq 5\right) \wedge\left(0 \leq x_{2} \leq 4\right) \wedge\left(0 \leq x_{3} \leq 4\right)\right\}$. The set of automaton

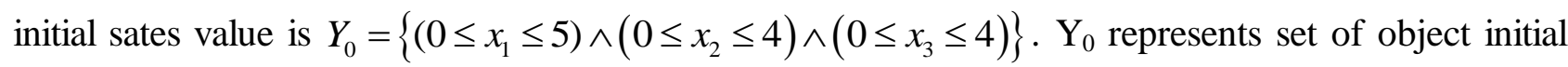
coordinates. $e x_{i}(i=1,2,3,4)$ includes discrete transition program $l a b_{i}(i=1,2,3,4)$. The set of discrete transition program is $L a b=\{l a b\}$. The discrete transition program which describes system discrete transition behaviour is $X^{\prime}=X$. In the discrete location $\mathrm{q}_{0}$ the three variables real time linear algebraic program $\mathrm{f}_{0}$ is $\left(\begin{array}{l}x_{1}^{\prime} \\ x_{2}^{\prime} \\ x_{3}^{\prime}\end{array}\right)=\left(\begin{array}{l}x_{1} \\ x_{2} \\ x_{3}\end{array}\right)+\left(\begin{array}{c}100 t \\ 100 t \\ 0\end{array}\right)$. In the discrete location $\mathrm{q}_{1}$ the three variables real time linear algebraic program $\mathrm{f}_{1}$ is $\left(\begin{array}{c}x_{1}^{\prime} \\ x_{2}^{\prime} \\ x_{3}^{\prime}\end{array}\right)=\left(\begin{array}{l}x_{1} \\ x_{2} \\ x_{3}\end{array}\right)+\left(\begin{array}{c}100 t \\ 100 t \\ 500 t\end{array}\right)$. In the discrete location $\mathrm{q}_{2}$ the three variables real time linear algebraic program $\mathrm{f}_{2}$ is $\left(\begin{array}{c}x_{1}^{\prime} \\ x_{2}^{\prime} \\ x_{3}^{\prime}\end{array}\right)=\left(\begin{array}{l}x_{1} \\ x_{2} \\ x_{3}\end{array}\right)+\left(\begin{array}{c}50 t \\ 50 t \\ -500 t\end{array}\right)$. In the discrete location $\mathrm{q}_{3}$ the three variables real time linear algebraic program $\mathrm{f}_{3}$ is $\left(\begin{array}{c}x_{1}^{\prime} \\ x_{2}^{\prime} \\ x_{3}^{\prime}\end{array}\right)=\left(\begin{array}{c}x_{1} \\ x_{2} \\ x_{3}\end{array}\right)+\left(\begin{array}{c}100 t \\ 100 t \\ -1000 t\end{array}\right)$. In the discrete location $\mathrm{q}_{4}$ the three variables real time linear algebraic program $\mathrm{f}_{4}$ is $\left(\begin{array}{c}x_{1}^{\prime} \\ x_{2}^{\prime} \\ x_{3}^{\prime}\end{array}\right)=\left(\begin{array}{c}x_{1} \\ x_{2} \\ x_{3}\end{array}\right)+\left(\begin{array}{c}50 t \\ 50 t \\ 1000 t\end{array}\right)$.

The invariants set $\operatorname{Inv}_{0}$ is

$\left\{\left(0 \leq x_{1} \leq 100\right) \wedge\left(0 \leq x_{2} \leq 100\right) \wedge\left(0 \leq x_{3} \leq 4\right)\right\}$.

The invariants set $\operatorname{Inv}_{1}$ is $\left\{\left(100 \leq x_{1} \leq 200\right) \wedge\left(100 \leq x_{2} \leq 200\right) \wedge\left(0 \leq x_{3} \leq 500\right)\right\}$.

The invariants set $\operatorname{Inv}_{2}$ is $\left\{\left(200 \leq x_{1} \leq 250\right) \wedge\left(200 \leq x_{2} \leq 250\right) \wedge\left(0 \leq x_{3} \leq 500\right)\right\}$.

The invariants set $\operatorname{Inv}_{3}$ is 
$\left\{\left(100 \leq x_{1} \leq 200\right) \wedge\left(100 \leq x_{2} \leq 200\right) \wedge\left(-1000 \leq x_{3} \leq 0\right)\right\}$.

The invariants set $\mathrm{Inv}_{4}$ is $\left\{\left(200 \leq x_{1} \leq 250\right) \wedge\left(200 \leq x_{2} \leq 250\right) \wedge\left(-1000 \leq x_{3} \leq 0\right)\right\}$.

The fighter flight movement automaton in all invariants set as a time section $\mathrm{T}=0.1$ seconds carries on three dimensional over approximate flow pipeline volume computation. Over approximate flow pipeline volume of fighter flight movement automaton are $\mathrm{V}_{0} \approx 11313, \mathrm{~V}_{1} \approx 41569, \mathrm{~V}_{2} \approx 40398, \mathrm{~V}_{3} \approx 80796$, $\mathrm{V}_{4} \approx 80199$.

\section{Summary}

In this paper, three dimensional over approximate flow pipeline volume algorithm of three variables real-time linear algebraic Hybrid Automaton is proposed. It can compute trajectory of three variables real-time linear algebraic Hybrid Automaton. In the future work, we will study its application.

\section{Acknowledgments}

This work is the final result of Introduction of Zhejiang University Of Media and Communications Scientific Research Grants Project(Z301B15521) and Zhejiang University Of Media and Communications Young Teacher Scientific Research Promotion Plan Project (ZC16XJ033).

\section{References}

[1] A. Chutinan and B. Krogh, "Verification of Polyhedral Invariant Hybrid Automata Using Polygonal Flow Pipe Approximation”, LNCS, vol. 1569, (1999), pp. 76-90.

[2] O. Botchkarev and S. Tripakis, "Verification of Hybrid Systems with Linear Differential Inclusion Using Ellipsoidal Approximations”, LNCS, vol. 1790, (2000), pp. 73-88.

[3] B. Allibert, "Analytic controllability of a linear hybrid system", SIAM Journal on Control and Optimization, vol. 37, no.3, (1999), pp. 844-868.

[4] S. Sankaranarayanan, H. Sipma and Z. Manna, "Non-Linear Loop Invariant Generation Using Groebner Bases", POPL04, (2004), pp. 318-329.

[5] F. David, G. Carlos and P. Miguel, "Coinductive Characterisations Reveal Nice Relations Between Preorders and Equivalences”, Electronic Notes in Theoretical Computer Science, vol. 212, (2008), pp. 149-162.

[6] H. Yang, J. Wu, Z. Zhang, "Approximate Completed Trace Equivalence of Inhomogeneous Linear Transition Systems", International Journal of Advancements in Computing Technology,vol. 4,no. 8,(2012), pp. 58-66.

[7] J. Fu, H. Tanner, J. Heinz and J. Chandlee, "Adaptive symbolic control for finite-state transition systems with grammatical inference", IEEE Transactions on Automatic Control. vol.59, no.2, (2014), pp. 505-511.

[8] V. Cheval, V. Cortier and S. Delaune, "Deciding equivalence-based properties using constraint solving", Theoretical Computer Science, vol. 492,(2013), pp. 1-39.

[9] M. Shen, "Hinfin filtering of continuous Markov jump linear system with partly known Markov modes and transition probabilities", Journal of the Franklin Institute, vol. 350, no. 10, (2013), pp. 3384-3399.

[10] H. Yang, J. Wu, Z. Zhang and Y. Liu, 2013 "Approximate Completed Trace Equivalence of Three Dimensional t-Model Nonlinear Algebraic Hybrid Systems", Applied Mathematics \& Information Sciences,vol.7, no.5, (2013), pp.1693-1697. 